\title{
Poster: Comparing Vibro-tactile Feedback Modes for Collision Proximity Feedback in USAR Virtual Robot Teleoperation
}

\author{
Paulo G. de Barros \\ Worcester Polytechnic Institute \\ 100 Institute Road \\ Worcester, MA, USA \\ $+1508-831-6617$
}

\author{
Robert W. Lindeman \\ Worcester Polytechnic Institute \\ 100 Institute Road \\ Worcester, MA, USA \\ $+1508-831-6712$
}

\begin{abstract}
Although multi-sensorial interfaces have been shown to improve user experience in different settings in Virtual Reality, these interfaces are not yet fully explored in urban search-and-rescue robot teleoperation. This paper presents a study on the performance effects of adding different types of vibro-tactile collision proximity feedback to a virtual robot's interface during a search task in a virtual environment. Results indicate that the addition of vibro-tactile feedback causes positive performance effects, especially for the intensity variation mode. Nevertheless, it also has a comfort impact for prolonged use.
\end{abstract}

KeYwords: Multi-sensorial displays, multi-modal interfaces, vibro-tactile feedback, tactors, robot-teleoperation, urban searchand-rescue

IndeX TeRms: H.5.2 [User Interfaces]: Haptic I/O, Evaluation/methodology; H.5.1 [Multimedia Information Systems]: Artificial, augmented and virtual realities; I.2.9 [Robotics]: Operator interfaces.

\section{INTRODUCTION}

The area of teleoperated urban search-and-rescue (USAR) robotics has significantly grown in popularity in recent years. In order to help robot interface research advance, it is critical to understand the usefulness of mappings between types of sensed data and the types of notifications available in different display devices, so that each piece of information is correctly conveyed to the operator without hindering the detection of other notifications and the performance of the main search task itself. Understanding such mappings is the main focus of this research.

The current work builds on previous result [1], and aims to evaluate the impact on performance when the robot output is enhanced with vibro-tactile feedback displays for robot collision avoidance in a search task. Two vibro-tactile interfaces, a vibration intensity variation mode and a vibratory pulse frequency mode, were compared to a no-vibration control case.

\section{RELATED WORK}

Specific interface design and implementation guidelines have yet to be standardized. Nevertheless, some progress has been made [1]. Input devices generally consist of keyboard, mouse, and joystick.

Data representation plays an important role in determining how

Paulo G. de Barros,pgb@wpi.edu

Robert W. Lindeman, gogo@wpi.edu

IEEE Symposium on 3D User Interfaces 2012

4-5 March, Orange County, CA, USA

978-1-4673-1205-9/12/\$31.00 @2012 IEEE difficult a USAR interface is to use. If the interface is too cognitively demanding, an operator may not succeed in both controlling the robot and completing his mission. Cognitive load variations have been previously claimed to affect the human-robot interaction (HRI) system performance due in part to variations in operator's situation awareness (SA) [3].

Although current USAR interfaces aim at improving SA and efficiency [5], little effort has been put on reducing cognitive load. The absence of operator-centered USAR-specific multisensorial interaction and interruption management evaluation studies indicates that such interfaces are still not fully explored. The work presented here attempts to attack the operator cognitive load problem through the use of feedback with multi-sensorial devices in addition to graphical ones, specifically vibro-tactile feedback.

\section{ROBOT INTERFACE}

The robot interface design 1 is similar to the one from our previous study [1]. It attempts to follow current USAR guidelines and is based on the work of Nielsen [5].

Our previous work [1] compared a visual and a vibro-tactile data display, both of which outperformed the control case when presented together but not when presented separately. In this work, we aim to compare different representations of the collision proximity information as vibro-tactile feedback only. This feedback is provided through a TactaBelt vibro-tactile interface [4].

Two vibro-tactile feedback modes are explored. In the first one (Intensity, or I), the closer the robot is to colliding in the direction the tactor points, the more intense a tactor in the TactaBelt continuously vibrates. In the second mode (Frequency, or F), the more frequently a tactor vibration pulsates or "beeps", the closer the robot is to colliding in the direction the tactor points. The latter mode differs from the former one because the vibration is not continuous. In both modes, the vibro-tactile feedback is only activated when the robot is within a distance $d$ of an object $(d \leq$ $1.25 \mathrm{~m})$. The operator controlled the robot using a Sony PlayStation2 Dual-shock joystick. The world was viewed through a standard 15.4" LCD screen at a resolution of $1024 \times 768$ at 17 fps.

\section{HYPOTHESES}

Based on our previous experiment and pilot study results, we hypothesize that:

H1. Using either vibro-tactile feedback interface should lead to an improvement in performance in the search task compared to the control case;

$\mathrm{H} 2$. Using the Intensity interface should lead to a higher performance improvement compared to the Frequency interface because of its easiness of use.

\section{EMPIRICAL STUDY}

A search task was designed to best reproduce what happens in reality in USAR situations, but in a simpler manner. Subjects had 
to search for red spheres (radius: $0.25 \mathrm{~m}$ ) in a debris-filled virtual environment (VE). They were asked to find as many spheres as possible in as little time as possible and also avoid the robot colliding with the VE. Twelve spheres were hidden, but subjects were unaware of the number. When the experiment was over, subjects drew a sketchmap of the VE showing the spheres found.

The experiment consisted of a within-subjects design. The independent variable (IV) was the type of collision-proximity feedback (CPF) interface, which included the interfaces I and $\mathrm{F}$ and a control case without vibro-tactile feedback ("None" or "N"). Interface presentation order in trials for each subject was partially balanced using Latin Square. In addition, the VE presentation order was also randomized.

Subjects had to wear the belt, even for the control case. Pictures taken using the robot camera were displayed on a web page with $800 \times 640$ pixels to help them during sketchmap drawing.

The dependent variables (DVs) were the time taken to complete the search task, the number of collisions, the number of spheres found, the number of collisions per minute, path length, the ratio between number of collisions and path length, the number of spheres found per minute, the ratio between number of spheres found and path length, and the quality of the sketchmaps.

Each subject took at most two hours to complete the study with some subjects completing it in only one hour. Subject gender and age, how often they played video games and used or worked with robots was collected in the demographics questionnaire. The spatial aptitude paper test had nine questions to be answered in five minutes, including painted-cube-face association and maporientation questions. A training session followed each trial so that subjects felt comfortable with the robot controls and to ensure they understood the TactaBelt behavior. In each treatment questionnaire, subjects had to draw sketchmaps, report the number of spheres found and answer questions about their levels of presence and comfort using the interfaces. It was based on the SUS and SSQ questionnaires. A final questionnaire comparing the three interfaces was also answered at the end of the experiment.

The experiment and training VEs as well as the robot interface were built using the $\mathrm{C} 4$ game engine (www.terathon.com). According to the AAAI Rescue Robotics Competition classification, the experiment VE has difficulty level yellow.

\section{RESULTS}

A total of 14 female and 22 male university students participated in the study (mean age: 19.67, S.D.: 1.49). The results were processed using a single-factor ANOVA with confidence level of $\alpha=0.05$. Close to significant results were described as trends $(\alpha=0.1)$. If a statistically significant difference (SSD) among more than two groups was found, a Tukey test (HSD, 95\% confidence level) was performed to reveal the groups that differed from each other.

Two important results were found for the dependent variables. Firstly, there was a clear improvement in number of collisions caused by both interfaces using vibro-tactile feedback. This was noticed in all dependent variables related to the number of collisions. This result not only confirmed our first hypothesis (H1), but also validates the soldity of our data analysis. Nonetheless, no distinguishing results for the Intensity and Frequency interfaces was found here to support our second hypothesis $(\mathrm{H} 2)$.

The second important result was the trend pointing to a slight decrease in the quality of map sketches for the Frequency interface compared to the other two interfaces. This may be a sign that a more advanced type of vibro-tactile feeback, such as the Frequency, is negatively impacting subejcts' cognitive load. The results for the treatment questionnaires were processed using the Friedman test for group comparisons and the Wilcoxon Exact
Signed-Rank test for pair-wise comparisons. On the one hand, both CPF interfaces have improved subject experience in the VE by increases in the "Being There" and "Visited" variables. On the other hand, both CPF interfaces had a lower rating than the control interface for the "Nausea" variable. In addition, the Frequency interface had a lower "Reality" rating than the other two.

For the final questionnaire, the results point to an increase in discomfort and distraction when using either CPF interfaces, with a larger increase caused by the Frequency interface. Furthermore, the Frequency interface appears to have also caused an increase in difficulty. Such a result was not detected in the treatment questionnaire data. These results seem to once again support $\mathrm{H} 2$.

\section{DISCUSSION}

Intensity and Frequency interfaces were proposed using as a basis the work by Nielsen et al. [5]. Our hypotheses claimed these interfaces would enhance subject performance $(\mathrm{H} 1)$ and that the Intensity interface would outperform the Frequency interface (H2).

Both of the proposed hypotheses were validated by the results obtained at least for part of the dependent variables considered. The lack of statistical strength in the sphere-finding results might have been due to learning effects.

In terms of $\mathrm{H} 2$, although no objective data supported it, the subjective data collected by both questionnaires did provide evidence for subjects' preference for the Intensity interface.

The above results together with the results of the previous experiment [1] lead us to believe that the use of vibro-tactile feedback interfaces enhances performance, especially when supplemented by data display through other senses.

\section{CONCLUSION}

To our knowledge, the present results for USAR robot teleoperation interfaces are novel. They offer an insight into how vibro-tactile feedback integrates in robot teleoperation and contribute as evidence of the benefits of vibro-tactile displays when designing multi-sensorial interfaces for USAR and perhaps other interface types. Future work consists of developing an adjustable USAR interface framework that allows the easy configuration of multi-sensorial interfaces. This framework will be built on USARSim. Further information about the current status of this research project can be found in [2].

\section{ACKNOWLEDGMENTS}

We thank the WPI Computer Science Department for their support.

\section{REFERENCES}

[1] de Barros, P.G., Lindeman, R.W., Ward, M.O. 2011. Enhancing robot teleoperator situation awareness and performance using vibrotactile and graphical feedback. Proceedings of IEEE 2011 Symposium on 3D User Interfaces. 3DUI'11, 47-54.

[2] De Barros, P.G., Professional Homepage, URL: http://users.wpi.edu/ ppg.

[3] M.R. Endsley, D.G. Garland. Theoretical Underpinning of Situation Awareness: A Critical Review. Situation Awareness Analysis and Measurement, Lawrence Erlbaum, Mahwah, NJ, 2000.

[4] Lindeman, R.W., Yanagida, Y., Noma, H., and Hosaka, K. 2006. Wearable vibrotactile systems for virtual contact and information display. Virtual Reality, Special Issue on Haptic Interfaces and Applications, 9, 2-3, 203-213, 2006.

[5] C.W. Nielsen, M.A. Goodrich, B. Ricks. Ecological interfaces for improving mobile robot teleoperation. IEEE Transactions on Robotics, volume 23, number 5, pages 927-941, October 2007. 